\title{
(6) OPEN ACCESS \\ Exploring the human factors of prescribing errors in paediatric intensive care units
}

\author{
Adam Sutherland, ${ }^{\oplus 1,2,3}$ Darren M Ashcroft, ${ }^{1,3}$ Denham L Phipps ${ }^{1,3}$
}

\begin{abstract}
${ }^{1}$ Centre for
Pharmacoepidemiology and Drug Safety, Division of Pharmacy and Optometry, School of Health Sciences, Faculty of Biology, Medicine and Health, The University of Manchester, Manchester, UK 2Paediatric Intensive Care Unit, Royal Manchester Children's Hospital, Manchester University NHS Foundation Trust, Manchester, UK

${ }^{3}$ NIHR Greater Manchester Patient Safety Translational Research Centre, University of Manchester, Manchester Academic Health Science Centre (MAHSC), Manchester, UK
\end{abstract}

\section{Correspondence to}

Adam Sutherland, Division of Pharmacy and Optometry, The University of Manchester, Manchester M13 9PL, UK; adam.sutherland@manchester. ac.uk

Received 3 August 2018 Revised 26 October 2018 Accepted 11 December 2018 Published Online First 8 February 2019
Check for updates

(C) Author(s) (or their employer(s)) 2019. Re-use permitted under CC BY-NC. No commercial re-use. See rights and permissions. Published by BMJ.

To cite: Sutherland A, Ashcroft DM, Phipps DL. Arch Dis Child

2019;104:588-595.

\begin{abstract}
Objective To explore the factors contributing to prescribing error in paediatric intensive care units (PICUs) using a human factors approach based on Reason's theory of error causation to support planning of interventions to mitigate slips and lapses, rules-based mistakes and knowledge-based mistakes.

Methods A hierarchical task analysis (HTA) of prescribing was conducted using documentary analysis. Eleven semistructured interviews with prescribers were conducted using vignettes and were analysed using template analysis. Contributory factors were identified through the interviews and were related to tasks in the HTA by an expert panel involving a PICU clinician, nurse and pharmacist.
\end{abstract}

Results Prescribing in PICU is composed of 30 subtasks. Our findings indicate that cognitive burden was the main contributory factor of prescribing error. This manifested in two ways: physical, associated with fatigue, distraction and interruption, and poor information transfer; and psychological, related to inexperience, changing workload and insufficient decision support information. Physical burden was associated with errors of omission or selection; psychological burden was linked to errors related to a lack of knowledge and/or awareness. Social control through nursing staff was the only identified control step. This control was dysfunctional at times as nurses were part of an informal mechanism to support decision making, was ineffective.

Conclusions Cognitive burden on prescribers is the principal latent factor contributing to prescribing error. This research suggests that interventions relating to skill mix, and communication and presentation of information may be effective at mitigating rule and knowledgebased mistakes. Mitigating fatigue and standardising procedures may minimise slips and lapses.

\section{BACKGROUND}

Approximately 237 million medication errors occur in the NHS in England every year, 66 million of which are clinically significant. ${ }^{1}$ These have been estimated to cost the National Health Service $£ 750 \mathrm{~m}$ per year. ${ }^{2}$ Children are twice as likely as adults to suffer harm as a result of medication error. ${ }^{3}$ The incidence of prescribing errors in paediatric intensive care unit (PICU) has been estimated at $11.1 \%-18 \%$ of prescriptions. ${ }^{45}$ However, to date, the increased vulnerability to harm has not been reflected in the representation of children's healthcare in medication safety research, which remains limited. Across a range of healthcare settings, electronic prescribing, standardisation of medication processes and doses and education are the only

\section{What is already known on this topic?}

- Prescribing error in paediatric intensive care is a common event and is associated with patient harm.

- These errors are multifactorial and associated with diverse contextual factors.

- Prescribing errors committed by junior doctors are often associated with psychological precursors, inadequate defences and line management deficiencies.

\section{What this study adds?}

Established human factors approaches and qualitative methods provide rich understanding of factors that contribute to prescribing error, suggesting interventions that may be useful in future research.

- The complexity of the contributory factors to prescribing error suggests that a single intervention is unlikely to be effective in reducing errors.

- Bundles of theory-based interventions are required to address factors that contribute to prescribing errors.

interventions to have demonstrated an effect on the incidence of medication errors ${ }^{6}$; the reasons for this, though, are poorly understood. ${ }^{7}$ In paediatric medicine in particular, previous studies have offered little insight into the factors that contribute to prescribing errors and so might usefully be targeted by improvement interventions. ${ }^{7}$ Those factors that have been suggested to date were inferred mainly from epidemiological studies, which provide only a description of event types and frequency, with no appreciation of the complex behavioural, cognitive and conceptual factors that also contribute to errors. In other areas of healthcare, human factors approaches have been used to explain the occurrence of patient safety incidents more broadly by taking into account the psychological, physical and organisational context of healthcare work. ${ }^{89}$ Given the insights obtained from these studies, it would be valuable to consider the application of similar methods to paediatric medication safety.

This study aimed to explore the factors that contribute to prescribing errors in PICU, in order to provide a theoretical basis on which interventions can be planned and evaluated. 


\section{METHOD}

The sampling frame and setting was healthcare professionals involved in medication prescribing in two PICUs in the north of England. The study was delivered in three phases: (1) the development of a hierarchical task analysis (HTA); (2) semi-structured interviews with prescribers; and (3) the merging of the interview data with the HTA in a Systematic Human Error reduction and Prediction Approach (SHERPA) to identify the failure modes and points and relating them to tasks within the HTA.

\section{Hierarchical task analysis}

HTA is a systematic method for analysing complex processes in terms of the behaviour that is involved. ${ }^{10}$ In HTA, the process starts with a defined goal (eg, generating a correct prescription) and identifies its constituent task steps. Each step is, in effect, a subgoal that must be achieved in order to meet the overall goal. The order of these subgoals is referred to as the plan. The task steps can be elaborated using the SHERPA. ${ }^{11}$ In order to suggest errors that may occur during task execution, SHERPA applies a taxonomy to each step according to the behaviour required to complete it: action; planning; evaluation; selection; checking; information retrieval; information entry; information communication; and calculation. The HTA was informed by documentary analysis of all formal materials relating to medicines prescribing, including prescription and administration charts, and guidelines and policies at both PICU sites. A document was only included if it was ratified via organisational governance processes, had appropriate document control and was within its expiry date. The key prescribing tasks were extracted from these documents and organised into an HTA using a proprietary software platform (Human Factors Workbench Version 3.7, Human Reliability Preston, Lancs 2010) by the first author (AS). The HTA was then reviewed by a human factors specialist (DLP) and subject matter experts from each centre, chosen to represent the main professions involved in medicines in PICU (two intensivists, two nurses and one pharmacist).

\section{Interviews}

A purposive sampling strategy was used to obtain sufficient representation of the staff roles involved in paediatric medicines prescribing at each study site. In total, there were 52 eligible participants across the two sites: 22 junior medical staff (paediatricians, anaesthetists, emergency medicine trainees and PICU trainees); 22 consultants; and 8 advanced nurse practitioners (ANPs). Participant recruitment was undertaken over 10 months (May 2016-February 2017). Potential participants were approached via email and through face-to-face contact during working hours. The characteristics of participants are presented in table 1 . The characteristics of the participating units are described in table 2 .

The semistructured interviews were carried out by the lead researcher (AS), a PICU pharmacist with experience in qualitative research. In total, 11 prescribers consented to participate in the study. These were six junior doctors, three consultants and two ANPs. Informed consent for participation was obtained. Interviews were conducted on a one-to-one basis in a private area of the PICU. In order to stimulate discussion during the interview, the interviewer presented three case vignettes that are outlined in box 1 . These vignettes were anonymised cases extracted from the patient safety incident database at one of the participating sites and represented each of the active failures in Reason's model of human error (skill-based errors (slipsand-lapses), rules-based mistakes [RBMs] and knowledge-based

\begin{tabular}{|ll|}
\hline Table 1 & Description of participants \\
\hline Participant reference & Profession \\
\hline Site 1 & \\
A1 & Consultant intensivist (also joined the SHERPA session) \\
A2 & Paediatric trainee \\
\hline A3 & Anaesthetic trainee \\
A4 & PICU trainee \\
A5 & Anaesthetic trainee \\
Site 2 & \\
\hline R1 & Advanced nurse practitioner \\
R2 & PICU trainee \\
\hline R3 & Consultant intensivist \\
\hline R4 & Advanced nurse practitioner \\
\hline R5 & Consultant intensivist \\
R6 & Paediatric trainee \\
SHERPA session & \\
\hline E1 & PICU research nurse \\
\hline E2 & Consultant intensivist \\
E3 & PICU pharmacist \\
\hline
\end{tabular}

PICU, paediatric intensive care unit; SHERPA, Systematic Human Error reduction and Prediction Approach.

mistakes $[\mathrm{KBMs}]) .{ }^{8}$ They provided contextual information for participants to relate to the situation and understand the event without explicitly suggesting any particular cause. Participants were asked to reflect on the vignettes sequentially and relate these to their own practice if possible.

Interviews were facilitated using a topic guide adapted from the London Protocol. ${ }^{12}$ The London Protocol is derived from Reason's "Swiss Cheese" model and designed to capture the contributing factors and context around a specific patient safety incident. The topic guide was structured to explore the complexity of the task being considered, the context within which the task was being undertaken (factors relating to the environment, the team, individual, the patient and the organisation) and participant's attitudes to these factors and the error being described.

Interviews were audio-recorded and transcribed verbatim. The transcripts were organised using version 11 of the NVivo qualitative analysis software (QSR International, 2016). Data were organised and analysed using template analysis. ${ }^{13}$ Template analysis takes large amounts of textual data and allows the researcher to structure and categorise these data at the outset, using a priori codes and themes that were identified from previous studies. ${ }^{12} 14$ These themes and categories are then adapted as analysis continues until the final analytical framework is produced. Data were coded initially by the lead researcher (AS) and independently by a second member of the research team (DLP). The template as prepared from the literature did not change through the analysis. Further subanalyses of prescribers by background, and by unit, was carried out to explore differences between professional background and unit

\begin{tabular}{lll} 
Table 2 & Characteristics of participating sites \\
& PICU1 & PICU2 \\
\hline Admissions/year & 850 & 750 \\
Full time equivalents consultants & 12 & 10 \\
Cardiac surgery & Yes & No \\
Prescribing system & Paper based & Paper based \\
\hline
\end{tabular}

$\mathrm{PICU}$, paediatric intensive care unit. 


\section{Box 1 Outline of case vignettes}

\section{Case 1 (slip and lapse type mistake)}

Error: missed prescription for anti-epileptic drug on discharge resulting in more frequent seizures.

Contextual factors: hurried discharge; intensive care unit prescriber was distracted a number of times.

\section{Case 2 (rules-based mistake)}

Error: ketamine prescribed for a postoperative patient requiring high dependency unit care without any indication, following unclear handover. Anaesthetic doses of ketamine were prescribed instead of analgesia. No ketamine was administered to the patient.

Contextual factors: hospital policy required repeated transcription of drugs from one chart to another; infusions were not visually verified.

\section{Case 3 (knowledge-based mistake)}

Error: a patient weaning from sedation became haemodynamically unstable and was mistakenly diagnosed as experiencing sedation withdrawal. Intravenous clonidine increased from $2 \mu \mathrm{g} / \mathrm{kg} / \mathrm{hour}$ to $3 \mu \mathrm{g} / \mathrm{kg} / \mathrm{hour}$. Patient suffered from complete heart block. Contextual factors: relatively junior paediatric intensive care unit doctor working alone overnight; senior colleague was attending a sick patient elsewhere in the hospital.

practice. However, there were no differences between the groups. The findings of the interviews were then placed into context with the HTA through a SHERPA. ${ }^{15}$ This method is an established consensus risk management technique that involves a group discussion about the validity of the findings and also to explore how error types might be controlled. The members of this group are presented in table 1.

\section{RESULTS}

The HTA identified that the prescribing process consisted of 30 subtasks (figure 1). All tasks needed to be executed correctly in order for a correct prescription to be written. From the SHERPA analysis (table 3), the behaviours required to write a correct prescription were predominantly related to information retrieval, entry and communication. This suggests a substantial cognitive burden on those carrying out the task.

During the interviews, cognitive demand was indeed suggested to be the main contributory factor for most errors and was often attributed by participants to distractions and interruptions in the course of their work. There was a continuous need to manage and prioritise workload, and all prescribers reported frequent interruptions to their work flow. While each patient had a single nurse, prescribers were allocated responsibility for many patients in a shift. The demands from each patient and nurse were different, and the prescriber needed to manage those demands alongside their own tasks. One way of managing demand was to delegate prescribing to colleagues. However, this use of informal support structures appeared to not always be effective.

If they're prioritising, you've also got the fact that [the prescriber's] head may be on a number of things... but that means that their whole attention won't be focussed on the task at hand. Participant R3

... We had a new patient... wasn't my patient, so I was trying to be helpful trying to write the drug chart. And I ended up just having to put the chart down and say, I need to go and have a cup of coffee now. I can't do this anymore. Participant A2
The problems associated with interruptions and distractions were exacerbated by conflicts between the perceived priorities of prescribers and those of other care providers in the team. From the HTA, it was identified that there were no physical actions, diagnostic or evaluative requirements in the process of writing a correct prescription. Therefore, prescribing may be cognitively taxing and complex but without an immediate impact on patient care. Prescriptions in PICU were predominantly handwritten, and there was a general perception that prescribing was a low priority task where other more hands-on activities were more important. Another reason for time pressure on prescribing was the practice of transcribing information from one prescription chart to another, either on admission/discharge or when the prescription chart was full. As the chart was required in order to administer medicines or discharge a patient out of PICU, this often led to prescribers having to work in a hurry.

I tend to leave that [prescribing] to later on. Usually when children come in it's quite a high intensity situation with intubation, lines, inotropes et cetera. Participant A3

The transcribed prescriptions were a rush job on the discharge ...

the pressure is there to get the patient out [of ICU]. Participant R4

Prescription charts were frequently transcribed at night as their design meant the last possible dose was given before midnight. There was evidence that this task was deprioritised during the day because of the lack of urgency but that led to the task becoming urgent in the middle of the night when prescribers were tired. In fact, fatigue was often identified as a contributory factor of prescribing errors and made it even more challenging to manage workload, prioritise activities and balance other professional duties.

... Nurses will hand you a prescription at four o'clock in the morning and say 'This whole chart needs redoing' ... and then they'll show you the chart, with every box filled and no signatures because they've been waiting for it all day... it's a heart-sink. Participant A4

The lack of good quality information on medicines for paediatric patients was also identified as part of the cognitive burden of this process. Prescribers were often involved in errors due to a lack of tacit knowledge about dosing and administration that was not available in official reference sources used on the unit. The interface between PICU and other wards and departments was also poorly defined. The procedures and documentation systems in other care areas all contributed to prescribing error as prescribers in PICU tried to access information or follow procedures that were unwritten or unclear. Medication was seldom part of formal handover processes and prescribers often had to piece together information on drug therapy from a range of sources that lead to errors associated with selecting the wrong drugs, doses or concentrations.

So, doses of drugs will be prescribed. This team have a certain way that they like to do it in their heads and they will run the drug on their own concentrations, but they don't necessarily write it down anywhere. Participant A3

In addition, the resources available in PICU—-the guidelines and policies-are all intended to support safe and effective prescribing but are largely rule based, in that they serve as instructions on how to write a correct prescription. They provide little support for the knowledge-based behaviour that would occur in non-routine situations that require the use of pharmacological or pharmacokinetic knowledge. Furthermore, the information in these guidelines is often inaccessible. When fatigued or under stress, prescribers therefore often relied on their own memory and the experience of the team around them rather than referring directly to documented practice or to consultants. 


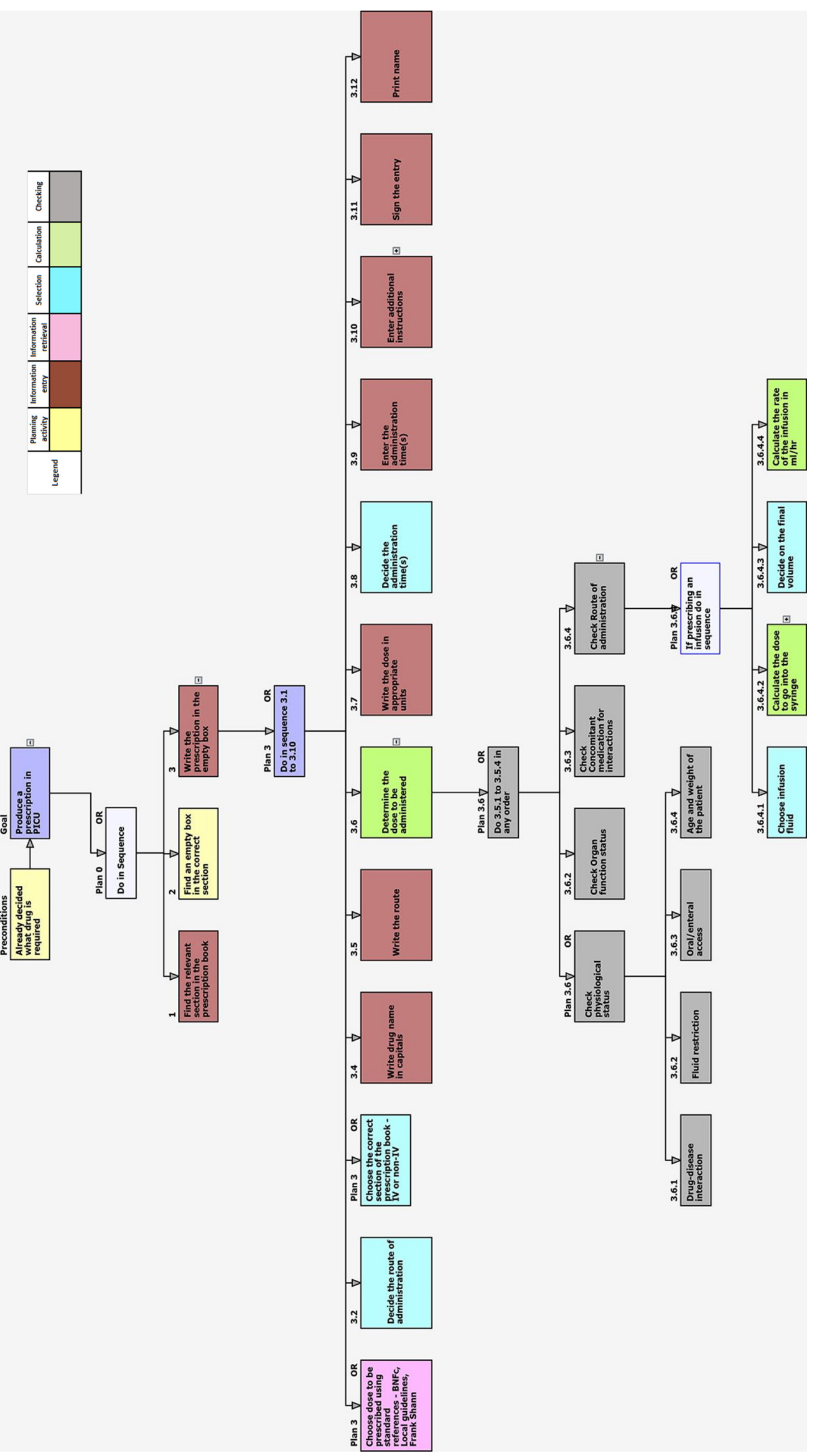

Figure 1 PICU, paediatric intensive care unit. 


\begin{tabular}{|c|c|c|}
\hline Theme & Categories & Examples \\
\hline Individual factors & $\begin{array}{l}\text { Inexperience. } \\
\text { Lack of skills or knowledge. }\end{array}$ & $\begin{array}{l}\text { Inexperienced in paediatric } \\
\text { prescribing (eg, an adult } \\
\text { anaesthetist on a short } \\
\text { rotation). }\end{array}$ \\
\hline Organisational factors & $\begin{array}{l}\text { Divergent practice and } \\
\text { systems. } \\
\text { Conflicting priorities. }\end{array}$ & $\begin{array}{l}\text { Different protocols between } \\
\text { patients receiving non- } \\
\text { intensive care unit (ICU) care } \\
\text { (postoperative recovery) and } \\
\text { those receiving ICU care. }\end{array}$ \\
\hline Task-related factors & $\begin{array}{l}\text { Ambiguous or inaccessible } \\
\text { information. } \\
\text { Reliance on memory and } \\
\text { previous experience. } \\
\text { Problem recognition. } \\
\text { Task complexity. }\end{array}$ & $\begin{array}{l}\text { Inability to locate guidelines } \\
\text { and procedures on the } \\
\text { intranet because of a lack of } \\
\text { computer facilities. }\end{array}$ \\
\hline Team-related factors & $\begin{array}{l}\text { Flat hierarchy. } \\
\text { Leadership and support. } \\
\text { Verbal communication. }\end{array}$ & $\begin{array}{l}\text { Lack of handover related to } \\
\text { medicines that the patient is } \\
\text { receiving on admission. }\end{array}$ \\
\hline Work-related factors & $\begin{array}{l}\text { Distraction and interruption. } \\
\text { Prioritisation. }\end{array}$ & $\begin{array}{l}\text { Rewriting a complex } \\
\text { medication chart and being } \\
\text { asked to review blood gases, } \\
\text { or answer the telephone. }\end{array}$ \\
\hline
\end{tabular}

When you open the guideline they're often very long and as you're scrolling down them ... you make sure you've got the right section. Participant R4

Interviewees referred to a flat hierarchy, where nursing staff were experienced practitioners and strong advocates for their patients. This could be intimidating to inexperienced prescribers who are likely to follow instruction rather than seek objective information about prescribing. It challenged anything that prescribers (particularly doctors) have experienced previously. The consultants were actively available to support junior prescribers; however, the latter were not always comfortable to ask for help because of a belief that they should be able to deal with the situation. This reluctance was short lived and once prescribers accepted their lack of experience and limited competency, and accepted the support of their consultants, the acceptance of help was described as 'liberating'.

And sometimes the doctors aren't aware that they don't know that much when it comes to these drugs ... and will go on the advice of the nurses. Participant A4

... As you become a bit more confident and a bit more senior, you ... realise that it's the consultants call. Participant A3

The results of the SHERPA discussion are presented in Table 4 including the identification of the controls of the error types and the potential mitigating factors for the future. This process identified variation in practice, distraction and interruption and fatigue as contributory factors in the occurrence of prescribing errors. It was identified that variation in practice may not be recognised as a problem within the leadership body of the units.

Everyone should be able to access information at the bedside about medicines and doses, surely?! Participant E2

Oh come on [name], that's a great idea in theory but everyone knows that you [the consultants] all have your preferred doses. Like dexamethasone for extubation - you don't want anyone extubated without it but [name] won't use it at all. It takes time for the guys to figure that out. Participant E1

It was also very clearly identified that the nurse was the only recovery step for many prescribing errors; however, the conflict of responsibilities of those nurses was also identified.
The nurses are so good at speaking up for their patients and making sure that tasks get done, but sometimes they forget that I as a doctor on the unit have six or seven other patients to look after as well. It's exhausting. Participant A1

Nurses want to help you when you're stuck, but sometimes we don't quite get it right, and if something isn't as we expect then we'll challenge it, even when we're wrong. Participant E2

This discussion also confirmed that the design of the PICU medication charts also predisposed to prescribing error.

It's so frustrating - the prescription chart gets full during the day shift, but it won't get rewritten until the middle of the night because that's when it can no longer be used. Participant E3

Well yes [name], but then we're trying to manage all the other stuff through the shift, and rewriting a prescription chart that can still be used doesn't get prioritised. Participant A1

\section{DISCUSSION}

Our findings present a rich understanding of both the process of prescribing in paediatric intensive care and the contributing factors to prescribing error in this field using a human factors approach. The key products of this study are the insights gained through integration of the HTA with qualitative interviews. The only other prescribing process in PICU in the literature was presented by Andrews et al and was a summary based on observed and reported medication errors in a single PICU and the opinion of the authors. ${ }^{16}$ It described the prescribing process in terms of actions only, with no consideration of the cognitive or behavioural elements of the process, thus presenting a more limited representation of the process than provided in the current study.

By using qualitative methods, we have also demonstrated evidence of the gap that exists between 'Work-as-imagined' (work that is laid out in official policies and procedures) and 'Work-as-done' (what has to be done in order to get the job done safely.) ${ }^{17}$ Additionally, we have been able to deepen our understanding of the contributory factors of prescribing errors in PICU. A key feature of prescribing in this setting is its cognitive burden, due to working in a fast-paced technical environment that is alien to most prescribers, which has also been identified as contributing to error in adult critical care. ${ }^{18}$ This cognitive burden is related to several characteristics of prescribing work in PICU: constant distraction and interruption; poor communication of important medicines-related information; constantly changing workload; a learning curve for newcomers; and lack of access to sufficient information to support decision making.

We have also identified that there are few controls in place to prevent prescribing errors in PICU (table 4). Reason identified three types of organisational control mechanisms to ensure safe function of systems in high-risk environments-administrative (rules, policies and procedures), social (cultural expectations of 'normal' behaviour) and self (a personal understanding of what is safe and what is not). ${ }^{19}$ We have identified that administrative controls are poorly designed and inaccessible, and lacking information to support knowledge-based behaviours. Thus, only social controls are available to mitigate prescribing errors through the intervention of nursing staff at the bedside. However, we have also shown that nurses are potentially implicated in the aetiology of prescribing error through propagation of organisational priorities and perpetuating distraction and interruption.

These findings have several implications when considering future interventions. Fatigue is of great interest in the medical and nursing literature, with tiredness and distraction identified as a leading contributory factor in medication errors among 


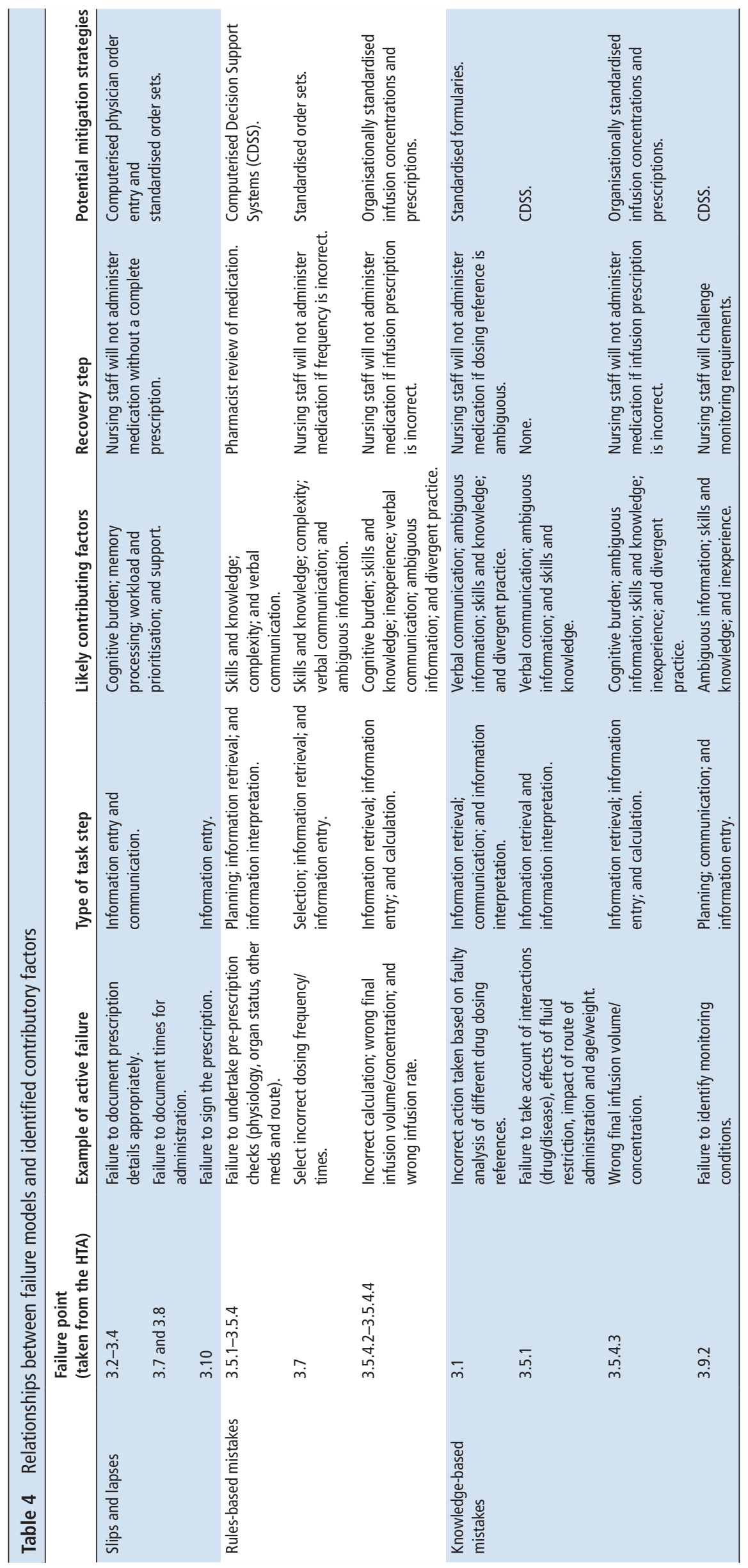


nurses. ${ }^{20} 21$ In other safety critical industries (notably aviation), there is a formal approach to the management of fatigue, which is lacking in medicine. ${ }^{22}{ }^{23}$ Thus, there is a need to develop systems for managing fatigue within the workforce, acknowledging that fatigue is physical and psychological.

With regards to the availability of information, it is well established that practice in healthcare is highly variable, often without any scientific basis for that variation. ${ }^{24}$ Therefore, consideration should be given to the impact of standardising medication prescribing resources. Computerised physician order entry (CPOE) with Computerised Decision Support Systems has been shown to be effective in reducing some medication errors. ${ }^{6}$ Standardisation of medication infusions has also been found to reduce prescribing errors in PICU. ${ }^{25}$ Evidence from other healthcare settings suggests that standardisation can reduce the reliance on team experience or leader interventions to ensure safe and effective practice, particularly in non-routine work situations. ${ }^{26}$ However, it is not a panacea; in practical terms, standardisation presents psychological and social challenges in its development and delivery that must be considered. ${ }^{27}$ From a philosophical perspective, it has been argued that to precisely and entirely describe a task is impossible, and therefore procedures are necessarily inadequate and incomplete. ${ }^{28}$ Hollnagel et al have identified that the adaptability of clinicians in situations of uncertainty is actually protective against error. ${ }^{17}$ Reason called this 'requisite imagination' ${ }^{19}$ where operators within a system can make use of prior experience, observations and underlying knowledge to critically evaluate options and make reasoned decisions where procedures are lacking. Correspondingly, we have identified that in periods of high stress there was a reliance on previous experience, or the experience of colleagues in the vicinity. The latter suggests that team dynamic and experience is an important resilience factor in PICU and may be as important as written guidelines. ${ }^{29}$ Team structure and team working in PICU is worthy of further comment in relation to prescribing. Many medical settings in the UK are hierarchical, and therefore doctors typically come into PICU being senior in their field and accustomed to making decisions. In this new environment, the balance is shifted, with responsibility for most decisions held by the consultant working in partnership with the wider healthcare team. An interesting finding in the current study is that the flat hierarchy in PICU may actually be a contributory factor for prescribing errors. Flat hierarchy has been reported in other studies to reduce error. ${ }^{30}$ However, this has been in the context of team orientation and organisational cultures where roles and tasks are well defined. In PICU, there is a diverse and well-trained workforce, but the role of the prescriber is vague and ill-defined and prey to the organisational demands of other team members and those outside of the team. Communication about medicines is erratic, and there can be a reticence among prescribers to ask for help in the early stages of their PICU assimilation. Therefore, we posit that interventions to improve team performance, including clear outlines of roles and responsibilities on induction to PICU, and adapting skill mix to reposition prescribing as a high priority, high-risk task may be beneficial to reduce prescribing errors. ${ }^{18} 31$

\section{Strengths and weaknesses of the research}

The use of qualitative methods within a human factors approach provides rich insights into the inherent weaknesses in PICU prescribing and the adaptations that prescribers have to make in order to prescribe safely. By involving consultants, non-medical prescribers and more senior medical staff, we have illuminated the contributory factors of prescribing errors in PICU setting. Our purposive sample included a range of professional backgrounds, and our subanalyses identified no differences in themes between any group. Furthermore, we are able to examine relationships between error types and the latent factors involved. However, the study also has some potential limitations. The use of case vignettes may have introduced some informant bias into the identification of contributory factors. ${ }^{32}$ A prospective contemporaneous interview methodology may have been better; however, this was not feasible with identification of errors and participants impossible in the study period. Additionally, this study did not reveal any unofficial reference sources being used in practice; however, the design of the study would have only detected and included institutionally sanctioned support materials. Future research involving direct observation may reveal other supplementary resources, if they exist. The potential for influence of the interviewer also cannot be discounted. As a PICU pharmacist, he had an intimate understanding of practice and prescribing errors. To mitigate this, he debriefed and reflected on the data regularly with a PICU research nurse. This study also focused on two PICUs that continue to use handwritten prescriptions; therefore, it may not be possible to generalise our findings into units that use CPOE.

\section{Conclusions}

PICU is a distracting and demanding environment, with prescribers juggling multiple tasks. Based on our findings, prescribing may be viewed as a low priority task with hands-on patient care perceived as more important. This contributes to a mental fatigue that is compounded by distraction and interruption, potentially leading to prescribing error. Patients are often outside the normal boundaries of care, but the guidelines prescribers are expected to adhere to are inadequately defined to protect patients, leading to RBMs and slips and lapses. KBMs are associated with a lack of direct training and education and the reliance on previous experience in order to prescribe medication. The flat hierarchy evident in PICU may be a short-term precursor for KBMs and RBMs. Prescribers traditionally rotate through PICU for relatively short periods and in their work outside of PICU are used to taking many decisions autonomously; thus, there is an adjustment period while prescribers acclimatise to the teamworking nature of PICU and they may not feel able to ask for help or advice from their consultants.

There are no functional administrative controls to mitigate prescribing errors in these PICUs resulting in very clear adaptations by prescribers in order to maintain performance. Single interventions to reduce prescribing error are unlikely to be effective as error types are interconnected, and thus cultural and behavioural interventions such as education and training, decision focused guidelines and greater use of multiprofessional working would be of benefit in this area.

Acknowledgements We would like to thank the medical and nursing staff of the two paediatric intensive care units (PICUs) involved in the study for their participation and cooperation in the data collection. In particular, we would like to thank Dr Lyvonne Tume, Dr Steve Kerr and Dr Mahil Samuel for enabling access to the study sites.

Contributors AS conceptualised and undertook study procedures under supervision of DMA and DLP. AS and DLP undertook data analysis. AS drafted the manuscript and both DMA and DLP have significantly contributed to all parts. All authors have had sight and agreement of the final manuscript.

Funding This study was funded through a National Institute for Health Research (NIHR)/Health Education England (HEE) Integrated Clinical Academic Training Programme MClinRes Studentship awarded to AS and supervised by DMA and DLP at the University of Manchester. This paper presents independent research funded by the NIHR and HEE.

Disclaimer The views expressed as those of the authors and not necessarily those of the NHS, the NIHR or the Department of Health. 
Competing interests None declared.

Patient consent Not required.

Ethics approval Ethical approval was granted by the University of Manchester Research Ethics Committee (UREC 15582).

Provenance and peer review Not commissioned; externally peer reviewed.

Data sharing statement There are no additional data associated with this study other than that reported.

Open access This is an open access article distributed in accordance with the Creative Commons Attribution Non Commercial (CC BY-NC 4.0) license, which permits others to distribute, remix, adapt, build upon this work non-commercially, and license their derivative works on different terms, provided the original work is properly cited, appropriate credit is given, any changes made indicated, and the use is non-commercial. See: http://creativecommons.org/licenses/by-nc/4.0/.

\section{REFERENCES}

1 Elliott R, Camacho E, Campbell F, et al. Prevalence and Economic Burden of Medicaton Errors in the NHS in England. Sheffield, UK, 2018.

2 National Reporting and Learning Service. Safety in doses. London, 2007.

3 Department of Health. National service framework for children, young people and maternity services: medicines for children and young people. London, 2007.

4 Cimino MA, Kirschbaum MS, Brodsky L, et al. Assessing medication prescribing errors in pediatric intensive care units. Pediatr Crit Care Med 2004;5:124-32.

5 Sutherland A, Talken-Sinclair J, Barber R. Reducing prescribing errors in a British PICU. Pediatr Crit Care Med 2011;12:A148.

6 Manias E, Kinney S, Cranswick N, et al. Interventions to reduce medication errors in pediatric intensive care. Ann Pharmacother 2014;48:1313-31.

7 Huynh C, Wong ICK, Correa-West J, et al. Paediatric patient safety and the need for aviation black box thinking to learn from and prevent medication errors. Paediatr Drugs 2017;19:99-105.

8 Reason J. Human error: models and management. BMJ 2000:320:768-70.

9 Holden RJ, Carayon P, Gurses AP, et al. SEIPS 2.0: a human factors framework for studying and improving the work of healthcare professionals and patients. Ergonomics 2013;56:1669-86.

10 Kirwan B, Ainsworth L. A guide to task analysis. 1st edn. London: Taylor Francis, 1992.

11 Embrey D. Qualitative and quantitative prediction of human error in risk assessment. I Chem E Symp Ser 1995;1:329-50.

12 Taylor-Adams S, Vincent C. Systems analysis of clinical incidents: the London protocol. London, 2000

13 King N. Using templates in the thematic analysis of text. In: Cassell C, Symon G, eds. Essential guide to qualitative methods in organisational research. London: SAGE Publications Ltd, 2004:256-70.
14 Dean B, Schachter M, Vincent C, et al. Causes of prescribing errors in hospital inpatients: a prospective study. Lancet 2002;359:1373-8.

15 Hughes CM, Baber C, Bienkiewicz M, et al. The application of SHERPA (Systematic Human Error Reduction and Prediction Approach) in the development of compensatory cognitive rehabilitation strategies for stroke patients with left and right brain damage. Ergonomics 2015;58:75-95.

16 Andrews C, Sutherland A, Fortune P. The impact of evidence-based interventions on reducing prescribing errors in PICU. Arch Dis Child 2014;99:e3-9.

17 Hollnagel E, Braithwaite J, Wears RL. Resilient health care: volume 2 : the resilience of everyday clinical work. 2015.

18 Reader TW, Flin R, Mearns K, et al. Developing a team performance framework for the intensive care unit. Crit Care Med 2009:37:1787-93.

19 Reason J, Parker D, Lawton R. Organizational controls and safety: the varieties of rulerelated behaviour. J Occup Organ Psychol 1998;71:289-304.

20 Flin R, Winter J, Sarac C, et al. Human factors in patient safety: review of topics and tools. Geneva 2009.

21 Khanade K, Sasangohar F. Stress, fatigue, and workload in intensive care nursing: a scoping literature review. Proc Hum Factors Ergon Soc Annu Meet 2017:61:686-90.

22 International Civil Aviation Organisation. Manual for the oversight of fatigue management approaches. Montreal, Canada, 2016.

23 Helmreich RL. On error management: lessons from aviation. BMJ 2000;320:781-5.

24 Lyons I, Furniss D, Blandford A, et al. Errors and discrepancies in the administration of intravenous infusions: a mixed methods multihospital observational study. BMJ Qual Saf 2018;27:892-901.

25 Arenas-López S, Stanley IM, Tunstell P, et al. Safe implementation of standard concentration infusions in paediatric intensive care. J Pharm Pharmacol 2017:69:529-36.

26 Künzle B, Zala-Mezö E, Kolbe M, et al. Substitutes for leadership in anaesthesia teams and their impact on leadership effectiveness. Eur J Work Organ Psychol 2010;19:505-31.

27 Wears RL. Standardisation and its discontents. Cogn Technol Work 2015;17:89-94.

28 Dekker $S$. Failure to adapt or adaptations that fail: contrasting models on procedures and safety. Appl Ergon 2003;34:233-8.

29 Bachrach DG, Lewis K, Kim Y, et al. Transactive memory systems in context: A meta-analytic examination of contextual factors in transactive memory systems development and team performance. J Appl Psychol 2018 [Epub ahead of print 19 Jul 2018].

30 Singer SJ, Falwell A, Gaba DM, et al. Identifying organizational cultures that promote patient safety. Health Care Manage Rev 2009;34:300-11.

31 Salas E, Cooke NJ, Rosen MA. On teams, teamwork, and team performance: discoveries and developments. Hum Factors 2008:50:540-7.

32 Gould D. Using vignettes to collect data for nursing research studies: how valid are the findings? J Clin Nurs 1996:5:207-12. 